\title{
Therapeutic effects of rituximab combined with cyclophosphamide on refractory idiopathic thrombocytopenic purpura
}

\author{
JIUHE WANG ${ }^{1}$, BIN WANG $^{2}$, ZHONGHUI SUN $^{3}$ and KEZENG XUE ${ }^{2}$ \\ ${ }^{1}$ Department of Hematology, Qingdao Hiser Medical Group; ${ }^{2}$ Ward Pharmacy, Qingdao Municipal Hospital; \\ ${ }^{3}$ Department of Pharmacy, The Eighth People's Hospital of Qingdao, Qingdao, Shandong 266000, P.R. China
}

Received June 11, 2018; Accepted January 16, 2019

DOI: 10.3892/etm.2019.7196

\begin{abstract}
Therapeutic effects of rituximab combined with cyclophosphamide on refractory idiopathic thrombocytopenic purpura (ITP) were investigated. We retrospectively analyzed 249 patients with refractory ITP who were admitted to Qingdao Hiser Medical Group between March 2013 and March 2017. Curative effects of patients treated with rituximab, cyclophosphamide, and combination therapy were observed and the changes of platelet count, PA IgG, and lymphocyte CD20 before and after treatment, as well as the incidence of adverse reactions after treatment, were compared. There was no significant difference in the expression of lymphocyte CD20, PA IgG and platelet count among the three groups of refractory ITP patients before treatment $(\mathrm{P}>0.05)$. After treatment, the expression levels of CD20 and PA IgG in lymphocytes were significantly downregulated, and platelet counts significantly increased in the three groups $(\mathrm{P}<0.05)$. After treatment, CD20 and PA IgG levels in combined therapy group were significantly lower, and platelet count was significantly higher, than those in the rituximab and cyclophosphamide groups $(\mathrm{P}<0.05)$. Also, after rituximab treatment, the expression levels of CD20 and PA IgG in lymphocytes were significantly lower than those in cyclophosphamide group $(\mathrm{P}<0.05)$, and platelet count was higher than that in cyclophosphamide group $(\mathrm{P}<0.05)$. After treatment, the total effective rate in combined therapy group was higher than that in the rituximab and cyclophosphamide group $(\mathrm{P}<0.05)$. Total effective rate of rituximab group was significantly higher than that of cyclophosphamide group $(\mathrm{P}<0.05)$. The incidence of adverse reactions in combined therapy group was $14.29 \%$ (12/84), which was significantly lower than that in cyclophosphamide group $(40.70 \%, 35 / 86$, $\mathrm{P}<0.05)$ and rituximab group $(29.11 \%, 23 / 79, \mathrm{P}<0.05)$. The application of rituximab combined with cyclophosphamide in
\end{abstract}

Correspondence to: Dr Kezeng Xue, Ward Pharmacy, Qingdao Municipal Hospital, 1 Jiaozhou Road, Qingdao, Shandong 266000, P.R. China

E-mail: kjf32v@163.com

Key words: rituximab, cyclophosphamide, combined therapy, refractory idiopathic thrombocytopenic purpura, efficacy the treatment of refractory ITP can improve patient's clinical symptoms. The efficacy of this technique is promising with no serious adverse reactions. This technique should be popularized in clinical practice.

\section{Introduction}

Idiopathic thrombocytopenic purpura (ITP) is a hemorrhagic disease, induced by the dysfunction of the immune system, which causes maturation disorder or thrombocytopenia. ITP is sometimes accompanied by an increase of bone marrow megakaryocytes and its incidence is relatively high in children (1). Clinical manifestations of ITP are chills, fever, and even sudden spontaneous mucosal bleeding in the skin (2). Non-specific treatment with large doses of $\gamma$-globulin, glucocorticoids, and splenectomy is effective in $70 \%$ of patients with ITP, while the efficacy in the remaining $30 \%$ of patients is low (3). Refractory ITP predominantly occurs in women, and high-dose hormonal drugs, such as immunosuppressant and $\gamma$-globulin, are frequently used. Immunosuppressive agents have a low rate of remission and may be associated with myelosuppression and secondary tumors. Remission rate of $\gamma$-globulin is high, as well as the cost, and the duration of curative effect is short (4).

In recent years, attention has been paid to the treatment of refractory ITP, and some new immunotherapy methods have been introduced (5). At present, cyclophosphamide and rituximab are frequently used in the treatment of patients with refractory ITP. Although the efficacy of cyclophosphamide in the treatment of refractory ITP patients is satisfactory, the incidence of adverse reactions, such as gastrointestinal reactions, bone marrow suppression, hair loss or even myocardial damage, is high (6). Rituximab can cause disorders, such as arrhythmias or hypotension (7). Martinez et al (8) have stated that the efficacy of rituximab in combination with cyclophosphamide in the treatment of patients with refractory ITP is superior to traditional single-agent therapy. However, studies on the efficacy and clinical significance of rituximab in combination with cyclophosphamide in the treatment of refractory ITP patients are relatively rare. In this study, clinical data of patients with refractory ITP were retrospectively analyzed to compare the treatment efficacies of the single and combined therapy. This study provides reference for the treatment of refractory ITP. 
Table I. Basic information of 249 patients with refractory ITP [n (\%)].

\begin{tabular}{|c|c|c|c|c|c|}
\hline Features & $\begin{array}{l}\text { Rituximab } \\
\text { group }(n=79)\end{array}$ & $\begin{array}{l}\text { Cyclophosphamide } \\
\text { group }(n=86)\end{array}$ & $\begin{array}{l}\text { Combined therapy } \\
\text { group }(n=84)\end{array}$ & $\chi^{2}$ test & P-value \\
\hline Sex & & & & 1.502 & 0.472 \\
\hline Male & $26(32.91)$ & $30(34.88)$ & $35(41.67)$ & & \\
\hline Female & $53(67.09)$ & $56(65.12)$ & $49(58.33)$ & & \\
\hline Age (years) & & & & 0.052 & 0.974 \\
\hline$<30$ & $42(53.16)$ & $47(54.65)$ & $46(54.76)$ & & \\
\hline$\geq 30$ & $37(46.84)$ & $39(45.35)$ & $38(45.24)$ & & \\
\hline Hematuria & & & & 0.905 & 0.636 \\
\hline Yes & $23(29.11)$ & $31(36.05)$ & $28(33.33)$ & & \\
\hline No & $56(70.89)$ & $55(63.95)$ & $56(66.67)$ & & \\
\hline \multicolumn{6}{|l|}{ Hematemesis or hemoptysis } \\
\hline Yes & $31(39.24)$ & $34(39.53)$ & $35(41.67)$ & 0.121 & 0.941 \\
\hline No & $48(60.76)$ & $52(60.47)$ & $49(58.33)$ & & \\
\hline Epistaxis & & & & 0.320 & 0.852 \\
\hline Yes & $46(58.23)$ & $50(58.14)$ & $52(61.90)$ & & \\
\hline No & $33(41.77)$ & $36(41.86)$ & $32(38.10)$ & & \\
\hline Platelet count (x 109/liter) & & & & 3.307 & 0.508 \\
\hline $20-50$ & $24(30.38)$ & 25 (29.07) & $30(35.71)$ & & \\
\hline$>50$ & $43(54.43)$ & $40(46.51)$ & $39(46.43)$ & & \\
\hline$<20$ & 12 (15.19) & $21(24.42)$ & 15 (17.86) & & \\
\hline
\end{tabular}

ITP, idiopathic thrombocytopenic purpura.

\section{Patients and methods}

Patient information. Clinical data of 249 patients with refractory ITP admitted to Qingdao Hiser Medical Group (Qingdao, China) from March 2013 to March 2017 were retrospectively analyzed. Among them, 79 patients were treated with rituximab, including 26 males and 53 females, aged 18-72 years, with a mean age of $31.54 \pm 4.78$ years; 86 patients were treated with cyclophosphamide, including 30 males and 56 females, aged $21-73$ years, with a mean age of $33.17 \pm 3.64$ years; and 84 patients were treated with both (combined therapy), including 35 males and 49 females, aged 18-72 years, with a mean age of $32.92 \pm 5.24$ years. No significant differences in the basic information were found among the three groups of patients (Table I).

Exclusion and inclusion criteria. Inclusion criteria: patients who met the refractory ITP diagnostic criteria (9); patients $>18$ years of age; patients who did not receive any relevant medical treatment in other hospitals. Exclusion criteria: patients who did not cooperate with the examination; patients with other secondary thrombocytopenia; patients with allergic reaction to the drugs used in the present study; patients during pregnancy and lactation; patients with acute gastrointestinal bleeding or other serious diseases; patients with communication and cognitive disorders. All participants or their family members signed an informed consent and cooperated with the medical staff to complete the relevant medical treatment.
The study was approved by the Ethics Committee of Qingdao Hiser Medical Group.

Methods. In rituximab group, $100 \mathrm{mg}$ of rituximab (state approval no. J20170034; Roche Pharmaceutical Co., Ltd., Shanghai, China) were dissolved in $100 \mathrm{ml}$ of saline and infusion was performed once a week at a rate of $50 \mathrm{ml} / \mathrm{h}$ for 4 weeks. Intravenous injection of $40 \mathrm{mg}$ of methylprednisolone (Pfizer Manufacturing Belgium NV, Puurs, Belgium) was performed to prevent related adverse reactions. In cyclophosphamide group, intravenous injection of cyclophosphamide (state approval no. H20093795; Yangtze River Pharmaceutical Group; Jiangsu HaiCi Biological Pharmaceutical Co., Ltd., Jiangsu, China) at a dose of $0.8 \mathrm{~g}$ was performed every week, and oral intake of cyclophosphamide at a dose of $2 \mathrm{mg} /(\mathrm{kg} \cdot$ day $)$ was performed every day, for a time period of 3 months. Both rituximab and cyclophosphamide were used in the combined treatment group using the same methods. Treatment was applied for 2 months. The curative effects of the three groups of patients were observed. Lymphocyte CD20 was detected by ELISA using the Human CD20 ELISA kit obtained from Jining Industrial Co., Ltd. (Shanghai, China). Platelet antibody IgG was detected using human anti-platelet antibody IgG kit purchased from Beyotime Institute of Biotechnology (Shanghai, China). Changes in platelet counts were monitored using Beckman Coulter DxH2400 hematology analyzer (Beckman Coulter, Inc., Brea, CA, USA). Occurrence of adverse reactions, such as dizziness or headache, nausea and vomiting, abnormal liver 
Table II. Lymphocyte CD20 before and after treatment in three groups of patients (\%).

\begin{tabular}{lccr}
\hline Groups & Before treatment & After treatment & t-test \\
\hline Rituximab group $(\mathrm{n}=79)$ & $28.6 \pm 3.5$ & $17.4 \pm 3.3$ & 20.69 \\
Cyclophosphamide group $(\mathrm{n}=86)$ & $27.8 \pm 3.9$ & $22.3 \pm 2.8^{\mathrm{a}}$ & $<0.001$ \\
Combined therapy group $(\mathrm{n}=84)$ & $28.1 \pm 3.7$ & $11.7 \pm 2.3^{\mathrm{a}, \mathrm{b}}$ & $<0.62$ \\
F-value & 0.954 & 50.71 & 34.5 \\
P-value & 0.387 & $<0.001$ & $<0.001$ \\
\hline
\end{tabular}

${ }^{\mathrm{a}} \mathrm{P}<0.05$, compared with rituximab group; ${ }^{\mathrm{b}} \mathrm{P}<0.05$, compared with cyclophosphamide group.

Table III. Changes of PA IgG before and after treatment in three groups of refractory patients (\%).

\begin{tabular}{lccr}
\hline Groups & Before treatment & After treatment & $\chi^{2}$ test \\
\hline Rituximab group $(\mathrm{n}=79)$ & $83 \pm 12$ & $46 \pm 12$ & 19.38 \\
Cyclophosphamide group $(\mathrm{n}=86)$ & $84 \pm 12$ & $50 \pm 13^{\mathrm{a}}$ & 17.82 \\
Combined therapy group $(\mathrm{n}=84)$ & $83 \pm 14$ & $33 \pm 9^{\mathrm{a}, \mathrm{b}}$ & $<0.001$ \\
F-value & 0.174 & 50.71 & $<0.001$ \\
P-value & 0.840 & $<0.001$ & $<0.001$ \\
\hline
\end{tabular}

${ }^{\mathrm{a}} \mathrm{P}<0.05$, compared with rituximab group; ${ }^{\mathrm{b}} \mathrm{P}<0.05$, compared with cyclophosphamide group.

and kidney function, and lung infection, after treatment were recorded.

Curative effects. Curative effects were evaluated according to relevant standards (10). Completely effective: if platelet count increases significantly to $>100 \times 10^{9} /$ liter, no bleeding occurs, and efficacy lasts $>2$ months. Partially effective: if platelet count increases significantly to $50-100 \times 10^{9} /$ liter, no bleeding basically occurs, and efficacy lasts $>2$ months. Minimal effective: when platelet count level fluctuates within 20-49×10 $/$ liter, bleeding symptoms are improved and duration is $>2$ months. Ineffective: if platelet count is $<20 \times 10^{9} /$ liter, no improvement or even worsening bleeding is observed. Total efficiency: completely effective + partially effective.

Statistical analysis. SPSS 19.1 software [AsiaAnalytics, Corp. (formerly SPSS China), Shanghai, China] was used for data processing. Measurement data, such as lymphocyte CD20, PA IgG, and platelet counts before and after treatment, were all expressed as mean \pm standard deviation. One-way analysis of variance was used to compare continuous data among multiple group,s with Dunnett's test used as post hoc test, and t-test was used for the comparison between two groups. Count data, such as patient basic data, efficacy evaluation, and incidence of adverse reactions were all expressed as rates, and comparisons between groups were analyzed using Chi-square test. $\mathrm{P}<0.05$ was considered to indicate a statistically significant difference.

\section{Results}

Changes of CD20 expression in lymphocytes of three groups of refractory ITP patients before and after treatment. There was no significant difference in the CD20 expression among the three groups of patients before treatment $(\mathrm{P}>0.05)$. After treatment, the CD20 expression levels were lower than those before treatment $(\mathrm{P}<0.05)$. Also, after treatment, CD20 expression level in combined therapy group was lower than that in rituximab and cyclophosphamide groups $(\mathrm{P}<0.05)$, and CD20 expression level in rituximab group was lower than that in cyclophosphamide group $(\mathrm{P}<0.05)$ (Table II).

Changes of PA IgG before and after treatment in three groups of refractory ITP patients. Before treatment, there was no significant difference in PA IgG among the three groups of refractory ITP patients $(\mathrm{P}>0.05)$. After treatment, there was a statistically significant difference in PA $\mathrm{IgG}$ among the three groups $(\mathrm{P}<0.05)$. PA IgG level was lower in combined therapy group than in rituximab and cyclophosphamide groups $(\mathrm{P}<0.05)$, and $\mathrm{PA}$ IgG level in rituximab group was lower than that in cyclophosphamide group $(\mathrm{P}<0.05)$ (Table III).

Changes of platelet count before and after treatment in three groups of refractory ITP patients. There was no significant difference in platelet counts among the three groups of refractory ITP patients before treatment $(\mathrm{P}>0.05)$. After treatment, the platelet counts of the three groups were higher than those before treatment $(\mathrm{P}<0.05)$. Platelet counts were higher in combined therapy group than in rituximab and cyclophosphamide groups $(\mathrm{P}<0.05)$, and platelet counts were also higher in rituximab group than that in cyclophosphamide group $(\mathrm{P}<0.05)$ (Table IV).

Comparison of curative effects after treatment in three groups of patients. After treatment, the total effective rate in 
Table IV. Platelet counts before and after treatment in three groups of patients (x10 $/$ liter).

\begin{tabular}{lccr}
\hline Groups & Before treatment & After treatment & t-test \\
\hline Rituximab group $(\mathrm{n}=79)$ & $12.3 \pm 1.7$ & $75.3 \pm 8.4$ & 65.34 \\
Cyclophosphamide group $(\mathrm{n}=86)$ & $12.6 \pm 1.3$ & $62.3 \pm 7.0^{\mathrm{a}}$ & $<0.001$ \\
Combined therapy group $(\mathrm{n}=84)$ & $12.5 \pm 1.4$ & $92.3 \pm 9.8^{\mathrm{a}, \mathrm{b}}$ & 64.74 \\
F-value & 0.882 & 267.9 & 73.88 \\
P-value & 0.415 & $<0.001$ & $<0.001$ \\
\hline
\end{tabular}

${ }^{\mathrm{a}} \mathrm{P}<0.05$, compared with rituximab group; ${ }^{\mathrm{b}} \mathrm{P}<0.05$, compared with cyclophosphamide group.

Table V. Comparison of curative effects after treatment in three groups of patients [n (\%)].

\begin{tabular}{|c|c|c|c|c|c|}
\hline Groups & $\begin{array}{l}\text { Completely } \\
\text { effective }\end{array}$ & $\begin{array}{l}\text { Partially } \\
\text { effective }\end{array}$ & $\begin{array}{l}\text { Minimal } \\
\text { effective }\end{array}$ & Ineffective & $\begin{array}{c}\text { Total effective } \\
\text { rate }\end{array}$ \\
\hline Rituximab group $(n=79)$ & $33(41.77)$ & $25(31.65)$ & $14(17.72)$ & $7(8.86)$ & $58(73.42)$ \\
\hline Cyclophosphamide group $(n=86)$ & $13(15.12)^{\mathrm{a}}$ & $36(41.86)$ & $23(26.74)$ & $14(16.28)$ & $49(56.98)^{\mathrm{a}}$ \\
\hline Combined therapy group $(n=84)$ & $58(69.05)^{\mathrm{a}, \mathrm{b}}$ & $17(20.24)^{b}$ & $7(8.33)^{b}$ & $2(2.38)^{b}$ & $75(89.29)^{\mathrm{a}, \mathrm{b}}$ \\
\hline F-value & 50.820 & 9.241 & 9.901 & 9.810 & 0.226 \\
\hline P-value & $<0.001$ & 0.010 & 0.007 & 0.007 & $<0.001$ \\
\hline
\end{tabular}

${ }^{\mathrm{a}} \mathrm{P}<0.05$, compared with rituximab group; ${ }^{\mathrm{b}} \mathrm{P}<0.05$, compared with cyclophosphamide group.

Table VI. Comparison of the incidence of adverse reactions after treatment in the three groups of patients [n (\%)].

\begin{tabular}{lccrr}
\hline Adverse reactions & $\begin{array}{c}\text { Rituximab } \\
\text { group }(\mathrm{n}=79)\end{array}$ & $\begin{array}{c}\text { Cyclophosphamide } \\
\text { group }(\mathrm{n}=86)\end{array}$ & $\begin{array}{c}\text { Combined therapy } \\
\text { group }(\mathrm{n}=84)\end{array}$ & $\begin{array}{c}\chi^{2} \text { test } \\
\text { P-value }\end{array}$ \\
\hline Dizziness or headache & $8(10.13)$ & $12(13.95)$ & $5(5.95)$ & 3.013 \\
Vomit & $12(15.19)$ & $15(17.44)$ & $5(7.14)$ & 0.222 \\
Liver and kidney dysfunction & $2(2.53)$ & $5(5.81)$ & $1(1.19)$ & 0.117 \\
Lung infection & $1(1.27)$ & $3(3.49)$ & $12(14.29)$ & 3.094 \\
Incidence of adverse reactions & $23(29.11)$ & $35(40.70)$ & 0.213 \\
\hline
\end{tabular}

combined therapy group was higher than that in rituximab and cyclophosphamide groups $(\mathrm{P}<0.05)$. Total effective rate in the rituximab group was higher than that in cyclophosphamide group $(\mathrm{P}<0.05)($ Table $\mathrm{V})$.

Comparison of the incidence of adverse reactions after treatment in the three groups of patients. After treatment, the incidence of adverse reactions in the three groups was significantly different from each other $(\mathrm{P}<0.05)$. Symptoms such as dizziness or headache, nausea, and vomiting were mostly self-relieving. In the rituximab group, 1 case of liver function abnormality, 1 case of renal function, and 1 case of lung infection occurred. In cyclophosphamide group, there were 2 cases of abnormal liver function, 3 cases of renal function, and 3 cases of pulmonary infection. In combined therapy group, there was 1 case of renal function, which was relieved after targeted treatment. No acute and late toxic reactions were observed (Table VI).

\section{Discussion}

ITP is a liquid immune-mediated systemic immune disease. In patients with ITP, platelet autoantibodies bind to platelet membrane glycoproteins and are phagocytosed by macrophages via Fc receptors (11). Treatment of ITP is aimed at reducing platelet destruction and controlling bleeding, while overtreatment should be avoided (12). The most common drug for the treatment of ITP is glucocorticoids, but some patients with ITP are insensitive or intolerant to glucocorticoids, which causes ITP patients to become refractory after glucocorticoid treatment (13). With the advancement of modern medicine, refractory ITP can be alleviated, but it is still difficult to achieve full rehabilitation, the incidence is high and the treatment cycle is long $(14,15)$. Therefore, the use of proper drugs for timely treatment is the key to control the deterioration of the disease (16).

Rituximab is a CD20 monoclonal antibody that binds to Fc receptors on abnormal $\mathrm{B}$ cells to initiate complement-mediated 
killing of cytotoxic antibodies. The killing effect leads to the solubilization of CD20 on B lymphocytes, inhibited production of antibodies by B lymphocytes, and reduced destruction of platelets. Rituximab has been widely used in the treatment of autoimmune hematological diseases (17). Cyclophosphamide can not only block the proliferation of B cells, but also inhibits the production of antibodies, which can be used to assist immunoadsorption therapy (18). Gödel et al (19) have shown that cyclophosphamide can treat autoimmune diseases by limiting the non-specific killing of small lymphocytes, inhibiting cell proliferation, and limiting the transformation of viral cells into immune mother cells. It is worth noting that cyclophosphamide interferes with the production of DNA and RNA and even cross-links with DNA, which in turn inhibits the immune response and proliferation and division of immune lymphocytes and impedes the deposition of immune complexes. Cyclophosphamide has been widely used in the treatment of lymphatic systemic and autoimmune diseases (20).

This study investigated the efficacy and clinical significance of rituximab in combination with cyclophosphamide for the treatment of refractory ITP patients. There was no significant difference in the expression levels of CD20 and PA IgG, and the platelet counts among the three groups of patients with refractory ITP before treatment $(\mathrm{P}>0.05)$. After treatment, the expression levels of CD20 and PA IgG in lymphocytes of three groups were lower than those before treatment, and the platelet counts were higher $(\mathrm{P}<0.05)$. After treatment, CD20 and PA IgG levels in combined therapy group were lower than those in rituximab and cyclophosphamide groups $(\mathrm{P}<0.05)$, while platelet count was higher $(\mathrm{P}<0.05)$. Also, after rituximab treatment, the expression levels of CD20 and PA IgG in lymphocytes were lower than those in cyclophosphamide group $(\mathrm{P}<0.05)$, while platelet count was higher $(\mathrm{P}<0.05)$. After treatment, total effective rate in combined therapy group was higher than that in rituximab and cyclophosphamide groups $(\mathrm{P}<0.05)$. Total effective rate of rituximab group was significantly higher than that of cyclophosphamide group $(\mathrm{P}<0.05)$. The data showed that the combined use of rituximab and cyclophosphamide intermittently results in the decrease of CD20 and PA IgG and increase of platelet count in some patients, which restores the abnormal immune function and significantly improves the quality of life. It has been reported (21) that rituximab is a human-mouse chimeric antibody that reduces the production of autoantibodies and the combination with cyclophosphamide can increase the immunosuppressive effect. In the present study, it was found that the incidence of adverse reactions in combined therapy group is $14.29 \%(12 / 84)$, which is significantly higher than that in cyclophosphamide group $(40.70 \%, 35 / 86, \mathrm{P}<0.05)$ and rituximab group $(29.11 \%, 23 / 79, \mathrm{P}<0.05)$. Symptoms such as dizziness or headache, nausea, and vomiting were mostly self-relieving. In the rituximab group, 1 case of liver function abnormality, 1 case of renal function, and 1 case of lung infection occurred. In cyclophosphamide group, there were 2 cases of abnormal liver function, 3 cases of renal function, and 3 cases of pulmonary infection. In combined therapy group, there was 1 case of renal function, which was relieved after targeted treatment. Al Askar et al (22) have shown that cyclophosphamide is toxic and combined treatment can reduce the dosage of cyclophosphamide, which in turn reduces the adverse reactions of cyclophosphamide and the damage to the body, and increase patient's compliance.

This study is limited by the small sample size. Future studies are required to confirm our conclusions.

In conclusion, application of rituximab combined with cyclophosphamide in the treatment of refractory ITP can improve patient's clinical symptoms. The efficacy of this technique is promising with no serious adverse reactions. This technique should be popularized in clinical practice.

\section{Acknowledgements}

Not applicable.

\section{Funding}

No funding was received.

\section{Availability of data and materials}

The datasets used and/or analyzed during the present study are available from the corresponding author on reasonable request.

\section{Authors' contributions}

JW collected and analyzed the general information of patients. BW and ZS performed ELISA. KX analyzed the curative effects. All authors read and approved the final manuscript.

\section{Ethics approval and consent to participate}

The study was approved by the Ethics Committee of Qingdao Hiser Medical Group (Qingdao, China). Patients who participated in this research, signed an informed consent and had complete clinical data. Signed informed consents were obtained from the patients or the guardians.

\section{Patient consent for publication}

Not applicable.

\section{Competing interests}

The authors declare that they have no competing interests.

\section{References}

1. Oved JH, Lee CS and Bussel JB: Treatment of children with persistent and chronic idiopathic thrombocytopenic purpura: 4 infusions of rituximab and three 4-day cycles of dexamethasone. J Pediatr 191: 225-231, 2017.

2. Takase K, Kada A, Iwasaki H, Yoshida I, Sawamura M, Yoshio N, Yoshida S, Iida H, Otsuka M, Takafuta T, et al: High-dose dexamethasone therapy as the initial treatment for idiopathic thrombocytopenic purpura: Protocol for a multicenter, open-label, single arm trial. Acta Med Okayama 72: 197-201, 2018.

3. Zhao CH and Hou M: Advances of research on abnormality of cell immunity in idiopathic thrombocytopenic purpura: Review. Zhongguo Shi Yan Xue Ye Xue Za Zhi 14: 1045-1048, 2006 (In Chinese).

4. Chandan JS, Thomas T, Lee S, Marshall T, Willis B, Nirantharakumar K and Gill P: The association between idiopathic thrombocytopenic purpura and cardiovascular disease: A retrospective cohort study. J Thromb Haemost 16: 474-480, 2018. 
5. Wasserman RL: Gammaplex ${ }^{\circledR} 5$ and $10 \%$ in the treatment of primary immunodeficiency and chronic immune thrombocytopenic purpura. Immunotherapy 9: 1071-1088, 2017.

6. Niscola P, Scaramucci L and Giovannini M: Spleen tyrosine kinase inhibition: A new promising approach to chronic and refractory immune thrombocytopenia. Immunotherapy 10: 5-7, 2018.

7. Vendramin C, Thomas M, Westwood JP, McGuckin S and Scully M: Rituximab-induced acute and delayed serum sickness in thrombotic thrombocytopenic purpura: The role of anti-rituximab antibodies. Br J Haematol: Mar 12, 2018 (Epub ahead of print). doi: 10.1111/bjh.15177.

8. Martinez AR, Paul MR and Kuo DJ: Treatment of immune thrombocytopenic purpura associated with cytomegalovirus infection in a child with pre-B cell acute lymphoblastic leukaemia after central nervous system relapse. BMJ Case Rep 2017: pii: bcr-2017-221947, 2017.

9. Page EE, Kremer Hovinga JA, Terrell DR, Vesely SK and George JN: Thrombotic thrombocytopenic purpura: Diagnostic criteria, clinical features, and long-term outcomes from 1995 through 2015. Blood Adv 1: 590-600, 2017.

10. Molineux G and Newland A: Development of romiplostim for the treatment of patients with chronic immune thrombocytopenia: From bench to bedside. Br J Haematol 150: 9-20, 2010.

11. Chowdhry M, Makroo RN, Thakur Y, Agrawal S, Mishra M and Rani D: Hyperheptaploidy in idiopathic thrombocytopenic purpura. Indian J Pathol Microbiol 61: 161-162, 2018.

12. Kubota M, Usami I, Kobayashi K, Tsutsui T and Matsubara K: Serum immunoglobulin levels at onset: Association with the prognosis of childhood idiopathic thrombocytopenic purpura. Int J Hematol 77: 304-307, 2003.

13. Raheja H, Kumar V, Hollander G, Shani J and Greenberg Y: Intravenous immunoglobulin-induced profound bradycardia in a patient with idiopathic thrombocytopenic purpura. Am J Ther 25: e572-e574, 2018.

14. Rezaeeyan H, Jaseb K, Alghasi A, Asnafi AA and Saki N: Association between gene polymorphisms and clinical features in idiopathic thrombocytopenic purpura patients. Blood Coagul Fibrinolysis 28: 617-622, 2017.
15. Peyvandi F, Scully M, Kremer Hovinga JA, Knöbl P, Cataland S, De Beuf K, Callewaert F, De Winter H and Zeldin RK: Caplacizumab reduces the frequency of major thromboembolic events, exacerbations and death in patients with acquired thrombotic thrombocytopenic purpura. J Thromb Haemost 15: 1448-1452, 2017.

16. Payandeh M, Sohrabi N, Zare ME, Kansestani AN and Hashemian AH: Platelet count response to Helicobacter pylori eradication in Iranian patients with idiopathic thrombocytopenic purpura. Mediterr J Hematol Infect Dis 4: e2012056, 2012.

17. Shoukat BA, Ali O, Kumar D, Bilal Gilani M, Zahid A, Aslam Joiya S and Anwar Malik M: Hypogammaglobulinemia observed one year after rituximab treatment for idiopathic thrombocytopenic purpura. Case Rep Med 2018: 2096186, 2018.

18. Warner NC, Vaughan LB and Wenzel RP: Human immunodeficiency virus associated thrombotic thrombocytopenic purpura, a clinical conundrum. J Clin Apher 32: 567-570, 2017.

19. Gödel P, Fischer J, Scheid C, Gathof BS, Wolf J and Rybniker J: Familial acquired thrombotic thrombocytopenic purpura in siblings - no immunogenetic link with associated human leucocyte antigens. Eur J Haematol 98: 311-313, 2017.

20. Rashid RM, Nabi Z, Ansari AZ and Qaiser QA: Immune thrombocytopenic purpura presenting in a patient after renal transplant for diabetic nephropathy. BMC Nephrol 19: 69, 2018.

21. Duperier T, Brody F, Felsher J, Walsh RM, Rosen M and Ponsky J: Predictive factors for successful laparoscopic splenectomy in patients with immune thrombocytopenic purpura. Arch Surg 139: 61-66, 2004.

22. Al Askar AS, Shaheen NA, Al Zahrani M, Al Otaibi MG, AlQahtani BS, Ahmed F, Al Zughaibi M, Kamran I, Mendoza MA and Khan A: Splenectomy vs. rituximab as a second-line therapy in immune thrombocytopenic purpura: A single center experience. Int J Hematol 107: 69-74, 2018.

(i) $\Theta$ This work is licensed under a Creative Commons Attribution-NonCommercial-NoDerivatives 4.0 International (CC BY-NC-ND 4.0) License. 